\title{
INFLUENCE OF DIMENSIONAL DEVIATIONS OF MECHANICAL ELEMENTS IN THE KINEMATIC STRUCTURE OF SCARA INDUSTRIAL ROBOTS ON POSITIONING PRECISION AND TRAJECTORY ACCURACY
}

\author{
ANDREI LUNCANU ${ }^{* 1}$, GHEORGHE STAN ${ }^{1}$ \\ 1 "Vasile Alecsandri" University of Bacau, Calea Marasesti 157, Bacau, 600115, Romania
}

\begin{abstract}
The purpose of this article is to present the research on the influence of the dimensional deviations of the mechanical elements in the kinematic structures of the SCARA industrial robots regarding the positioning precision and trajectory error. In this article the direct kinematics method is used to calculate the positioning of the end-effector and generate the trajectory simulation with and without dimensional deviations.
\end{abstract}

Keywords: SCARA industrial robot, trajectory tracking error, positioning error

\section{INTRODUCTION}

Regarding the predictions presented by the International Federation of Robotics [1] of SCARA industrial robot sales in the coming years, the number of SCARA industrial robots used is growing. Due to higher production rates and higher precisions of the industrial operations, new researches regarding development of methods to increase the positioning precision and trajectory tracking accuracy are needed. In my research regarding determining new methods to enhance industrial robot's trajectory tracking accuracy, I have come to a conclusion that most industrial robots control systems are using the automated trajectory planning system algorithm [2]. By using this algorithm without any compensating methods of the dimensional and geometric deviations present in a SCARA industrial robot structure, I have observed positioning and trajectory tracking errors. By researching I have determined that most of the positioning and trajectory tracking errors are caused by dimensional deviations of kinematic links linking element. The main purpose of this article is to present a mathematical method of modeling and simulation of the influences caused by this type of deviation. In order to determine the influences cause by the dimensional deviation, we need to determine the end-effector's positioning mathematical model. The mathematical model used for this article is the direct kinematic model with homologous transfer matrices used by other researchers [3-6]. In order to determine the influences caused by this type of deviation the direct kinematic model of the SCARA industrial robot with the dimensional deviations and the direct kinematic model of the SCARA industrial robot without the dimensional deviations. By comparing the SCARA industrial robot positioning equations with dimensional equations and without dimensional deviations we have determined that the positioning equations used to calculate the end-effector positioning and trajectory generating are different. Because of these differences the positioning and trajectory tracking accuracies are affected. By using the SCARA industrial robot's kinematic parameters and the values of dimensional deviations values of milling CNC machining tolerances found in STAS 8100/4-88 in a Matlab simulation method [7] based on the direct kinematic models of the SCARA industrial robots with and without dimensional deviations, we have determined the maximum position and trajectory tracking errors. As a result of researching the influence of dimensional deviations within industrial robot's structure the positioning and trajectory tracking accuracy can be permanently

\footnotetext{
Corresponding author, email: andreiluncanu@yahoo.com

(C) 2018 Alma Mater Publishing House
} 
enhanced due to the systematic characteristic of the dimensional deviation. Because the method is easy to use and uses generalist parameters of industrial robots it can be used for any type of industrial robot.

\section{DETERMINING THE INFLUENCE OF DIMENSIONAL DEVIATION IN THE KINEMATIC STRUCTURE OF SCARA INDUSTRIAL ROBOT REGARDING THE POSITION PRECISION AND TRAJECTORY TRACKING ACCURACY}

In order to determine the influence of dimensional deviations of the mechanical elements within the SCARA industrial robot's structure on the end-effector's positioning precision and trajectory tracking accuracy, the positioning equation of the end-effector mounted on the SCARA industrial robot are needed. The equations of the end-effector are obtained throw computing the direct kinematic model of the SCARA industrial robot. In order to determine the direct kinematic model, the kinematic structure of the SCARA industrial robot is needed and it is shown in Figure 1.

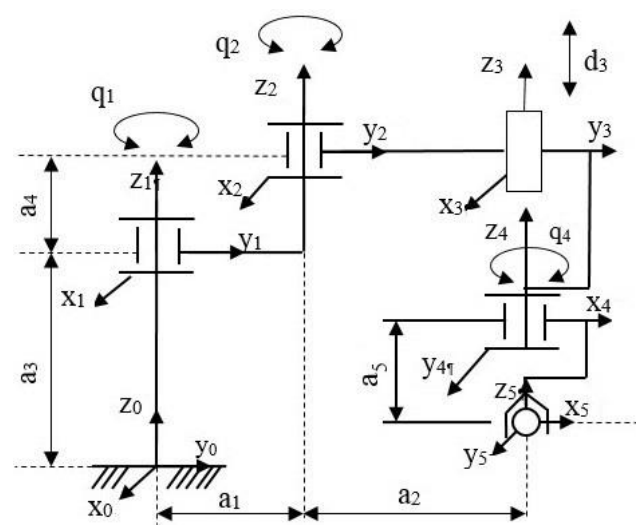

Fig. 1. The kinematics structure of SCARA industrial robot.

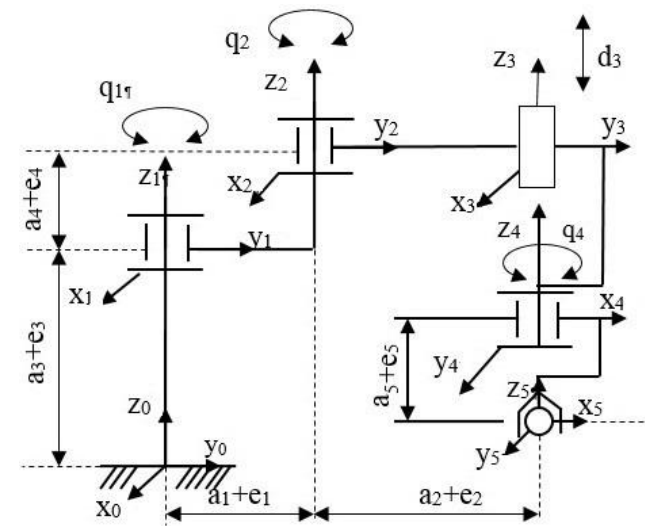

Fig. 2. The kinematic structure of the SCARA industrial robot with dimensional deviation.

By analyzing the kinematic structure of the SCARA industrial robot and by applying the direct kinematic modeling theory with homogenous transfer matrixes [3 - 6] the direct kinematic model of the SCARA industrial robot is obtained and it is presented in equation (1).

$$
\mathrm{T}_{05}=\mathrm{L}_{01} \cdot \mathrm{R}_{12} \cdot \mathrm{L}_{12} \cdot \mathrm{R}_{23} \cdot \mathrm{L}_{23} \cdot \mathrm{R}_{34} \cdot \mathrm{L}_{34} \cdot \mathrm{L}_{45}
$$

By substituting the elements from the direct kinematic model (1) with the homogeneous transfer matrices of the kinematic model. The computed matrix for the direct kinematic model $\mathrm{T}_{05}$ contains both the orientation equations and positioning equations of the end-effector. Because of the focus on the positioning and trajectory tracking accuracy, only the positioning equations from the resulted matrixes are needed and are shown in the system (2).

$$
\begin{gathered}
x=a_{2} \cdot\left(\cos \left(q_{1}\right) \cdot \cos \left(q_{2}\right)-\sin \left(q_{1}\right) \cdot \sin \left(q_{2}\right)\right)+a_{1} \cdot \cos \left(q_{1}\right) \\
y=a_{2} \cdot\left(\cos \left(q_{1}\right) \cdot \sin \left(q_{2}\right)+\cos \left(q_{2}\right) \cdot \sin \left(q_{1}\right)\right)+a_{1} \cdot \sin \left(q_{1}\right) \\
z=a_{3}+a_{4}+a_{5}+d_{3}
\end{gathered}
$$

As can be seen in the above equations, all dimensional and kinematic parameters in the kinematic structure of the SCARA industrial robot participate in the end-effector positioning and in the generation of the SCARA industrial robot trajectory.

Due to the machining tool precision of the mechanical elements used by the SCARA industrial robot structure, dimensional errors are produced and affect both the trajectory tracking accuracy and positioning precision of the SCARA industrial robots.

By analyzing the kinematic structure of the SCARA industrial robot and using the dimensional deviations the structure of the SCARA industrial robot with the dimensional deviations is obtained and it is shown in Figure 2. 
By applying the mathematical modeling theory on the new SCARA industrial robot kinematic structure the new direct kinematic model is obtained and it is shown in the equation (3).

$$
\mathrm{T}_{05}=\mathrm{L}_{01} \cdot \mathrm{L}_{01 \mathrm{e}} \cdot \mathrm{R}_{12} \cdot \mathrm{L}_{12 \mathrm{e}} \cdot \mathrm{L}_{12} \cdot \mathrm{R}_{23} \cdot \mathrm{L}_{23 \mathrm{e}} \cdot \mathrm{L}_{23} \cdot \mathrm{R}_{34} \cdot \mathrm{L}_{34} \cdot \mathrm{L}_{45 \mathrm{e}} \cdot \mathrm{L}_{45}
$$

By substituting the elements from the direct kinematic model (3) with the homogeneous transfer matrices of the kinematic model the end-effector positioning equations are computed and shown in the system (4).

$$
\begin{gathered}
\mathrm{x}=a_{2} \cdot\left(\cos \left(q_{1}\right) \cdot \cos \left(q_{2}\right)-\sin \left(q_{1}\right) \cdot \sin \left(q_{2}\right)\right)+e_{2} \cdot\left(\cos \left(q_{1}\right) \cdot \cos \left(q_{2}\right)-\sin \left(q_{1}\right) \cdot \sin \left(q_{2}\right)\right)+a_{1} \cdot \cos \left(q_{1}\right)+e_{1} \cdot \cos \left(q_{1}\right) \\
\mathrm{y}=a_{2} \cdot\left(\cos \left(q_{1}\right) \cdot \sin \left(q_{2}\right)+\cos \left(q_{2}\right) \cdot \sin \left(q_{1}\right)\right)+e_{2} \cdot\left(\cos \left(q_{1}\right) \cdot \sin \left(q_{2}\right)+\cos \left(q_{2}\right) \cdot \sin \left(q_{1}\right)\right)+a_{1} \cdot \sin \left(q_{1}\right)+e_{1} \cdot \sin \left(q_{1}\right) \\
\mathrm{z}=\mathrm{a}_{3}+\mathrm{a}_{4}+\mathrm{a}_{5}+\mathrm{d}_{4}+\mathrm{e}_{3}+\mathrm{e}_{4}+\mathrm{e}_{5}
\end{gathered}
$$

By comparing the equations of the end-effector positioning without and with dimensional deviations we can observe differences in all the positioning equations of the end-effector. According to the differences found both the positioning precision and trajectory tracking are affected.

\section{RESULTS AND DISCUSSION}

By using the direct kinematic model with dimensional deviations obtained throw computation in specialized simulation programs, the positioning errors and the trajectory tracking errors of the SCARA industrial robot can be determined. In order to obtain the best results for this simulation of the influence produced by the dimensional deviations, the dimensional parameters of the LS6 industrial robot produced by Epson will be used.
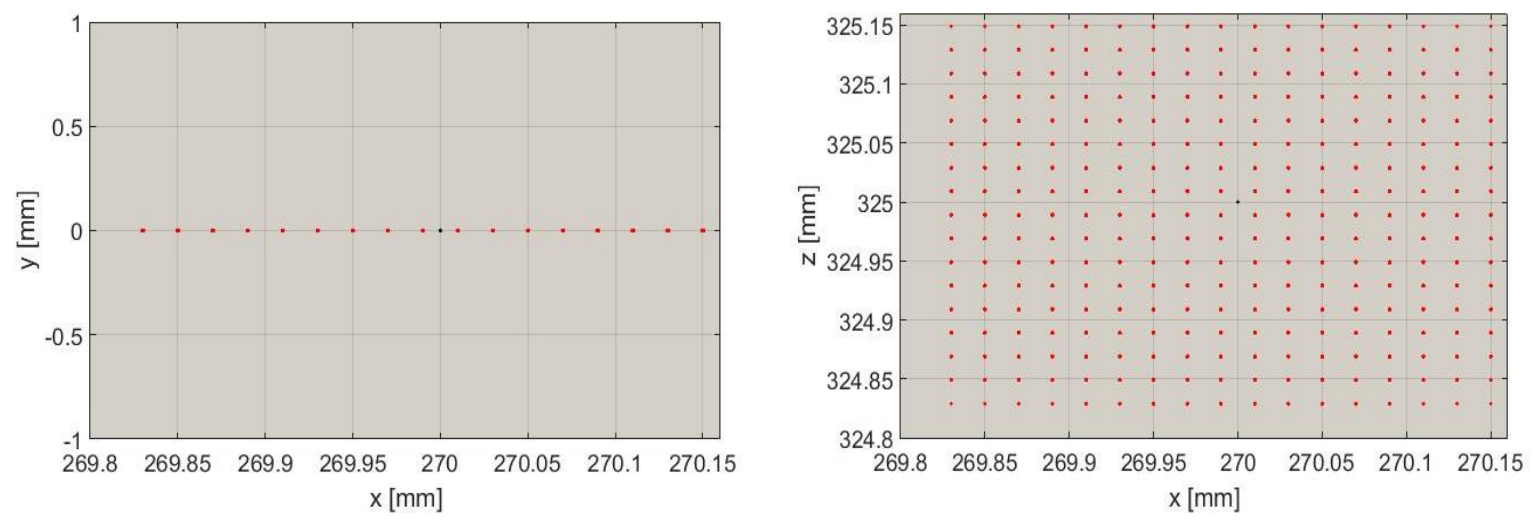

Fig. 3. Positioning error simulation by using the direct mathematical model with dimensional deviation.

These are $a_{1}=125 \mathrm{~mm}, a_{2}=145 \mathrm{~mm}, a_{3}=200 \mathrm{~mm}, a_{4}=50 \mathrm{~mm}$ and $a_{5}=75 \mathrm{~mm}$. According to the ISO tables with tolerances of mechanical machining for a milling machine the maximum values of dimensional deviations used for the LS6 SCARA robot are $\mathrm{e}_{1}$ between $-0.081 \mathrm{~mm}$ and $0.081 \mathrm{~mm}, \mathrm{e}_{2}$ between $-0.089 \mathrm{~mm}$ and $0.089 \mathrm{~mm}, \mathrm{e}_{3}$ between $-0.081 \mathrm{~mm}$ and $0.081 \mathrm{~mm}, \mathrm{e}_{4}$ between $-0.036 \mathrm{~mm}$ and $0.036 \mathrm{~mm}$ and $\mathrm{e}_{5}$ between -0.054 and 0.054 $\mathrm{mm}$.

By introducing the parameter for a positioning $\mathrm{q}_{1}=0^{\circ}, \mathrm{q}_{2}=0^{\circ}, \mathrm{d}_{3}=0 \mathrm{~mm}$ and $\mathrm{q}_{4}=0^{\circ}$, dimensional parameters of the LS6 SCARA industrial robot and the dimensional deviations in MATLAB [7] in order to simulate the positioning errors of the SCARA industrial robot, shown as in Figure 3.

As can be observed from the simulation the black dot is the theoretical position of the SCARA industrial robot. The red dots are the positioning errors of the SCARA industrial robot produced by the dimensional deviations.

By measuring the mechanical components and introducing corrections in the trajectory planning algorithm [2]. In order to determine the impact of dimensional deviations on the trajectory tracking accuracy a trajectory path must be constructed by using the displacements from Table 1. 
Table 1 Kinematic links displacement for trajectory.

\begin{tabular}{|c|c|c|c|c|}
\hline $\begin{array}{c}\text { Number of } \\
\text { displacement }\end{array}$ & $\mathrm{q} 1$ & $\mathrm{q} 2$ & $\mathrm{~d} 3$ & $\mathrm{q} 4$ \\
\hline 1. & -22 & 60 & 30 & -30 \\
\hline 2. & -21 & 61 & 31 & -29 \\
\hline 3. & -20 & 62 & 32 & -28 \\
\hline 4. & -19 & 63 & 33 & -27 \\
\hline 5. & -18 & 64 & 34 & -26 \\
\hline 6. & -17 & 65 & 35 & -25 \\
\hline
\end{tabular}

By using the displacement of the kinematic links from Table 1 in the direct kinematic model with dimensional deviations the simulation for the trajectory tracking accuracy of the SCARA industrial robot is shown Figure 4.

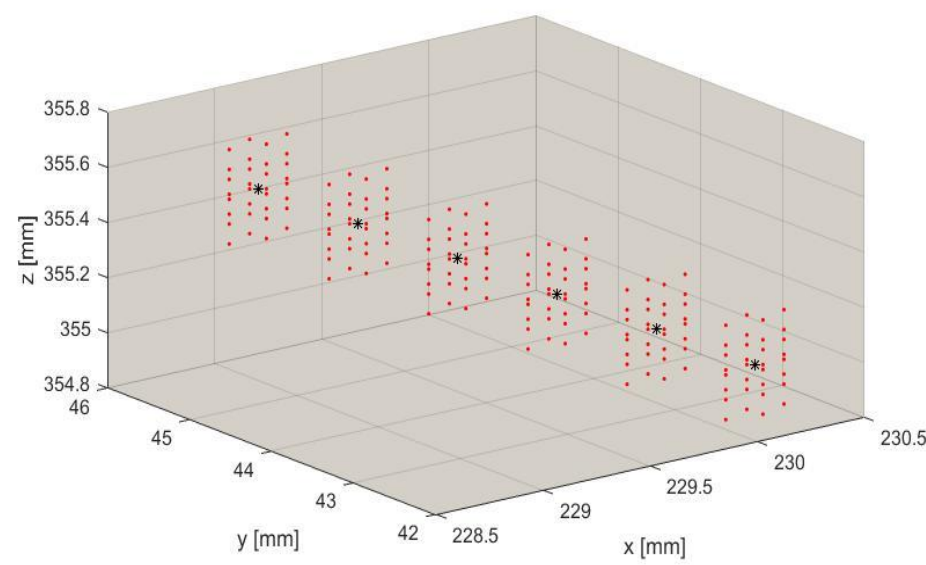

Fig. 4. Trajectory tracking error simulation by using the direct mathematical model with dimensional deviation.

As we can observe from Figure 4 the dimensional deviations can produce trajectory tracking errors. From Figure 3 it can be seen that the end-effector positioning errors are between $-0.2 \mathrm{~mm}$ and $0.2 \mathrm{~mm}$. The values of the positioning errors are higher than any other industrial robot found on the market and are higher for the demand of industrial operations positioning precision. By using the same program to simulate the trajectory with the displacements from table 1 the trajectory tracking errors are between $-0.2 \mathrm{~mm}$ and $+0.2 \mathrm{~mm}$.

\section{CONCLUSIONS}

As can be seen from the results of the simulations, the SCARA industrial robot is affected by the dimensional deviations present in the kinematic structure. By using the methods to determine the mathematic model of the SCARA industrial robot with dimensional deviations and using capable simulating programs as used in this article can improve the speed of determination of the impact of dimensional deviations on different types of industrial robots and develop new ways to compensate this type of deviation. As can be seen from the results extracted from the simulations, the maximum positioning and tracking error value are between $-0.2 \mathrm{~mm}$ and 0.2 $\mathrm{mm}$.

These values are too high for any type of positioning system and this is due of using the maximum manufacturing tolerances in the simulation. But in reality, the dimensional deviations are far lower due to the technological improvement in this field. By using special measurement systems with high precision and introducing compensating methods both the positioning and trajectory tracking accuracy can be permanently improved.

\section{REFERENCES}

[1] https://ifr.org/ifr-press-releases/news/world-robotics-report-2016, World Robotics Report 2016.

[2] Saravana Perumaal, S., Jawahar, N., Automated trajectory planner of industrial robot for pick-and-place task, International Journal of Advanced Robotic Systems, vol. 10, no. 100, 2013, p. 1-17. 
[3] Melchiorri, C., Kinematic Model of Robot Manipulators, Dipartimento di Ingegneria dell'Energia Elettrica e dell'Informazione (DEI), Universita di Bologna; anul 2014, on line http://wwwlar.deis.unibo.it/people/cmelchiorri/Files_Robotica/FIR_01_Industrial_Robotics.pdf.

[4] Kucuk, S., Zafer, B., Robot Kinematics: Forward and inverse kinematics, industrial-robotics-theorymodelling-control, 2006, p. 117- 148, on line http://cdn.intechweb.org/pdfs/379.pdf.

[5] Kumar, K.K, Srinath, A., Jugalanvesh, G., Premsai, P., Suresh, M., Kinematic analysis and simulation of a 6 DOF kuka KR5 robot for welding application, International Journal of Engineering Research and Applications, vol. 3, no. 2, 2013, p. 820-827.

[6] Gouasmi, M., Ouali, M., Fernini, B., Meghatria, M., Kinematic modelling and simulation of a 2-R robot using solidworks and verification by MATLAB/Simulink, International Journal of Advanced Robotic Systems, vol. 9, no. 245, 2012, p. 1- 13.

[7] Mustafa, A.M., AL-SAIF, A., Modeling, simulation and control of 2-R robot, Global Journal of Researches in Engineering Robotics \& Nano-Tech, vol. 14, no. 1, 2014, p. 58-54. 